\title{
Decompensated cirrhosis is the commonest presentation for NAFLD patients undergoing liver transplant assessment
}

\author{
Authors: Ahmed Hussain, ${ }^{A}$ Preya J Patel, ${ }^{B}$ Freya Rhodes, ${ }^{B}$ Ankur Srivastava, ${ }^{B}$ David Patch ${ }^{C}$ and William Rosenberg ${ }^{D}$
}

\begin{abstract}
Non-alcoholic fatty liver disease (NAFLD) accounts for $10-15 \%$ of orthotopic liver transplants (OLTs) in the UK. Index presentations with cirrhotic decompensation represent missed opportunities for preventive treatment leaving OLT or palliation as the only options.

We retrospectively reviewed patient records for all NAFLD patients undergoing assessment for OLT between January 2003 and December 2017.

Data were available for 81 patients with NAFLD as the primary diagnosis. Fifty-two patients had decompensated cirrhosis at first presentation; $91.7 \%$ presented to secondary care compared to $52.7 \%$ referred from primary care $(p=0.001)$. Cirrhosis was not suspected at the time of referral to hospital in $\mathbf{2 4 . 7 \%}$ of patients subsequently assessed for OLT.

Most patients undergoing assessment for OLT for NAFLD had decompensated cirrhosis at their first diagnosis of chronic liver disease. These data highlight the plight of patients with NAFLD cirrhosis in whom chronic liver disease is diagnosed late.
\end{abstract}

KEYWORDS: Non-alcoholic fatty liver disease, transplantation, decompensation, cirrhosis, prognosis

DOI: 10.7861/clinmed.2019-0250

\section{Introduction}

Non-alcoholic fatty liver disease (NAFLD) is the most common cause of chronic liver disease (CLD) in many developed countries and affects $20-30 \%$ of people in Europe and North America. ${ }^{1,2}$ Rising trends in obesity have resulted in a rise in the incidence of NAFLD and medical complications associated with fatty liver disease and metabolic syndrome. ${ }^{3}$ The presence of advanced fibrosis is associated with increased risks of overall and liver-related

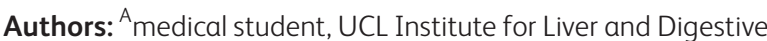
Health, London, UK; ${ }^{B}$ clinical research fellow, UCL Institute for Liver and Digestive Health, London, UK; ${ }^{C}$ consultant hepatologist, UCL Institute for Liver and Digestive Health, London, UK; ${ }^{D}$ professor of liver diseases, UCL Institute for Liver and Digestive Health, London, UK mortality. ${ }^{4,5}$ NAFLD is the most rapidly increasing aetiology on the liver transplant waiting list; a retrospective study in the USA found that the incidence of NAFLD on the liver transplant list had increased by $170 \%$ between 2004 and $2013 .{ }^{6}$ Over the past 5 years, NAFLD accounted for between $10-15 \%$ of patients listed annually for orthotopic liver transplants (OLTs) in the UK.?

An index presentation with hepatocellular cancer or a decompensating event, such as ascites, hepatic encephalopathy or variceal haemorrhage, has a profound impact on morbidity and mortality and has a significant negative impact on the patient's quality of life. ${ }^{8}$ Earlier detection of NAFLD provides opportunities to instigate interventions such as lifestyle modifications with the aim of sustained weight loss resulting in fibrosis regression, potentially averting or reducing the likelihood of the serious lifethreatening complications of portal hypertension. ${ }^{9-14}$ Furthermore, earlier detection of hepatocellular cancers creates possibilities for interventions with curative potential such as resection and radiofrequency ablation rather than transplantation or palliation that are often the only option with late diagnosis.

Despite the rising incidence and burden of NAFLD and its associated comorbidities, there remains a poor awareness regarding its recognition and management. Concerningly, the Veterans Administration primary care centre study highlighted that patients at highest risk of NAFLD were not being evaluated for this condition. ${ }^{15}$ Only $21.5 \%$ of patients who were identified by study investigations had a confirmed diagnosis of NAFLD in primary care, $14.7 \%$ were counselled regarding diet and exercise and $10.4 \%$ were referred to a specialist.

We hypothesise that many patients reaching liver transplant listing are only diagnosed with NAFLD at a point where liver disease has resulted in irreversible complications. We have conducted a retrospective analysis of patients with NAFLD cirrhosis referred for OLT assessment, aiming to determine their disease status at their first presentation to healthcare, and their subsequent clinical outcomes.

\section{Methods}

This was a cross-sectional analysis of all patients who underwent OLT assessment for a sole indication of NAFLD at the Royal Free London NHS Foundation Trust between January 2003 and December 2017. NAFLD was defined by the sonographic demonstration of hepatic steatosis in the presence of metabolic risk factors and the exclusion of significant alcoholic consumption 
or other causes of chronic liver disease (CLD) and was confirmed by reviewing the histology of the explant. ${ }^{16}$ This study (Integrated Research Application System number 270340) was evaluated using the Health Research Authority 'Is my study research?' tool and judged to not be considered research by the NHS, therefore no ethical approval or patient consent was required.

\section{Clinical Data}

Data was collected retrospectively by interrogating the electronic care records for all included patients; demographic, clinical, laboratory and outcome data were extracted. Prospective data on transplantation, and mortality for all included patients were recorded. Base hospitals and referring physicians were contacted to collate data regarding each patient's index presentation to healthcare. Patients were classified as 'decompensated' if they had an index presentation of jaundice, ascites, variceal bleeding, encephalopathy or hepatocellular carcinoma (HCC).

\section{Data analysis}

Participant sociodemographic and clinical characteristics were described using frequency and percentage for categorical variables, mean and standard deviation (SD) for continuous data normally distributed, and median and interquartile range (IQR) for non-normally distributed data. Normality was assessed using the Shapiro-Wilk test. The relationship between two categorical variables was assessed using Pearson's $\chi^{2}$ test or Fisher's exact test as appropriate. The comparison of a continuous variable between two groups was tested using independent t-tests or Mann-Whitney $U$ tests when the normality assumption was not met. KaplanMeier survival analysis was performed to assess the difference in survival between those patients with and without decompensated disease at their index presentation, and those who did and did not undergo transplantation. All $p$ values were two-sided and statistical significance was set at $\alpha=0.05$. Data analysis was conducted using SPSS version 23.0 (IBM Corp, Armonk, 2013).

\section{Results}

Eighty-one patients with a primary diagnosis of NAFLD underwent OLT assessment at the Royal Free London, a tertiary transplant centre between January 2003 and December 2017 (supplementary material S1). Fifty-seven patients entered healthcare through referral from a primary care physician to local secondary care services, while 24 patients presented directly to secondary care as emergency cases. Twenty-six patients were excluded from our initial pool of 107 patients due to incomplete data.

\section{Patient journey}

All of the 81 NAFLD patients who underwent OLT assessment had established cirrhosis at the time of their first diagnosis of CLD whether in primary or secondary care. For 20 (24.7\%) patients the diagnosis of cirrhosis was not recognised at the patients' first presentation to healthcare, this included 18 patients referred from primary care for the investigation of deranged liver function tests (LFTs). The remaining patients were diagnosed with cirrhosis at their first presentation to healthcare with a decompensating episode $(n=52)$ or when cirrhosis was incidentally detected on imaging $(n=9)$.

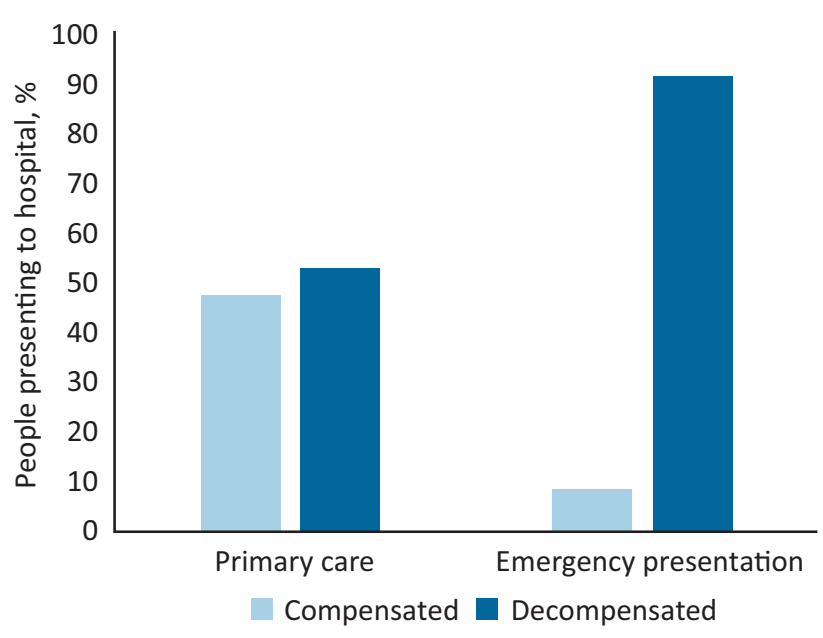

Fig 1. Proportions of patients presenting to healthcare with decompensated disease.

Fifty-two patients had decompensated cirrhosis at first presentation to healthcare with liver disease. Twenty-two patients classed as decompensated first encountered healthcare for CLD as an emergency, compared to the 30 referred by their primary care physician, this represented $91.7 \%$ of the patients who presented directly to secondary care $(p=0.001 ;$ Fig 1$)$. The most common reasons for referral/presentation to secondary care was the development of ascites $(n=28)$, abnormal LFTs $(n=18)$ or a variceal bleed ( $n=16$; supplementary material S2).

\section{Reason for referral to tertiary care}

At the time of referral to tertiary care for transplant assessment, 72 patients had a primary diagnosis of NAFLD cirrhosis. The others were referred with a diagnosis of 'cryptogenic cirrhosis' or cirrhosis of 'unknown aetiology'. Fifty-four patients were referred for OLT workup, while 15 patients were referred for a second opinion regarding the present suitability of OLT. Twelve patients were referred from secondary care to either optimise management or undergo procedures that could only be undertaken at the tertiary care centre; all were subsequently assessed for OLT (supplementary material S3).

The specific indication for referral to the transplant centre was only recorded in 66 patients' notes. Increasingly deranged LFTs were the sole reason for referral in three patients who eventually underwent OLT assessment. Of these 66 patients, 58 had evidence of decompensation including ascites $(n=29)$, encephalopathy $(n=17)$, variceal bleeding $(n=9)$ or HCC $(n=18)$. One patient was referred due to the development of hepatopulmonary syndrome. Thirteen $(30 \%)$ patients had more than one indication for referral listed in notes.

\section{Patient characteristics at first tertiary centre appointment}

Patient characteristics at their first attendance at a tertiary care appointment are summarised in Table 1. The only significant difference was that patients classed as decompensated had a higher prevalence of HCC $(p=0.041)$. Seventy patients had 
Table 1. Characteristics of patients at the first tertiary centre appointment $(n=81)$, stratified by index presentation of chronic liver disease

\begin{tabular}{|c|c|c|c|}
\hline & $\begin{array}{l}\text { Decompensated on initial } \\
\text { presentation to healthcare, } \\
\text { total } n=52\end{array}$ & $\begin{array}{l}\text { Compensated on initial } \\
\text { presentation to healthcare, } \\
\text { total } n=29\end{array}$ & $\mathrm{p}$ value \\
\hline Age, years, median (IQR) & $59.0(55.0-62.0)$ & $57.2(53.0-63.0)$ & $0.752^{a}$ \\
\hline Encephalopathy, n (\%) & $12(23.1)$ & $10(34.5)$ & $0.269^{b}$ \\
\hline Ascites, n (\%) & $33(63.5)$ & $14(48.3)$ & $0.184^{b}$ \\
\hline Varices, n (\%) & $19(36.5)$ & $11(37.9)$ & $0.901^{b}$ \\
\hline Hepatocellular carcinoma, n (\%) & $14(26.9)$ & $2(6.9)$ & $0.041^{c}$ \\
\hline Ischemic heart disease, $\mathrm{n}$ ( \%) & $3(5.8)$ & $1(3.4)$ & $>0.999^{c}$ \\
\hline Vascular disease, n (\%) & $3(5.8)$ & $1(3.4)$ & $>0.999^{c}$ \\
\hline Diabetes, n (\%) & $40(76.9)$ & $27(93.1)$ & $0.075^{c}$ \\
\hline Hypertension, n (\%) & $20(38.5)$ & $15(51.7)$ & $0.248^{b}$ \\
\hline Hypercholesterolaemia, n (\%) & $4(7.7)$ & $5(17.2)$ & $0.270^{c}$ \\
\hline Platelets, $\times 10^{9} / \mathrm{L}$, median (IQR) & $115.0(82.0-186.0)$ & $120.0(65.0-149.0)$ & $0.198^{a}$ \\
\hline International normalised ratio, median (IQR) & $1.3(1.1-1.4)$ & $1.4(1.2-1.5)$ & $0.280^{a}$ \\
\hline Sodium, mmol/L, median (IQR) & $139.0(136.0-141.5)$ & $139.5(137.0-143.5)$ & $0.514^{\mathrm{a}}$ \\
\hline Creatinine, $\mu \mathrm{mol} / \mathrm{L}$, median (IQR) & $84.0(67.0-118.5)$ & $83.5(68.5-110.5)$ & $0.766^{\mathrm{a}}$ \\
\hline Bilirubin, $\mu \mathrm{mol} / \mathrm{L}$, median (IQR) & $24.0(11.0-44.0)$ & $30.0(11.5-48.0)$ & $0.528^{a}$ \\
\hline Alanine aminotransferase, $U / L$, median (IQR) & $35.0(25.5-44.0)$ & $28.5(20.0-44.0)$ & $0.106^{a}$ \\
\hline Aspartate aminotransferase, U/L, median (IQR) & $46.0(31.0-59.5)$ & $46.0(35.5-58.0)$ & $0.562^{a}$ \\
\hline Fibrosis-4 score, mean \pm SD & $4.37 \pm 3.17$ & $5.60 \pm 3.03$ & $0.099^{d}$ \\
\hline Non-alcoholic fatty liver disease fibrosis score, mean \pm SD & $1.637 \pm 1.657$ & $2.371 \pm 1.370$ & $0.057^{d}$ \\
\hline UK Model for End-Stage Liver Disease score, mean \pm SD & $52.4 \pm 5.6$ & $53.4 \pm 6.1$ & $0.443^{d}$ \\
\hline Model for End-Stage Liver Disease score, median (IQR) & $13.0(10.0-17.0)$ & $16.0(11.5-18.0)$ & $0.111^{\mathrm{a}}$ \\
\hline
\end{tabular}

decompensated cirrhosis at their first tertiary centre appointment with $53 \%$ of patients exhibiting more than one sign of decompensation. Ascites was present in $58 \%$ of patients at the time of OLT assessment. Patients classed as decompensated had a median Charlson comorbidity index of 5 (IQR 4-6). Decompensated patients were significantly more likely to have a higher Charlson index $(p=0.039)$ than the compensated patients.

\section{Transplant listing and outcomes}

Of the 81 patients assessed for OLT for NAFLD, 59 patients were listed for transplant. Of these, only 43 patients underwent organ transplantation (Fig 2). Of those classed as decompensated at their index presentation, 31 patients underwent transplantation $(p=0.115)$. None of the patients transplanted had compensated disease at the time of OLT. Eight patients died post transplantation; four died within 6 months postoperatively from either graft or multi-organ failure. Three patients died on the waiting list without transplantation. Two patients recovered liver function sufficiently to be removed from the waiting list and two patients subsequently refused transplantation after listing.
One patient was de-listed due to positive blood alcohol levels. The remaining eight were de-listed due to their HCC progressing beyond transplant criteria $(n=2)$ or the patients' had deteriorated and they were deemed unfit for transplantation $(n=6)$.

Of the 22 patients considered unsuitable for transplantation, $15(68.2 \%)$ had comorbidities that precluded transplantation. These contraindications included poor pulmonary function $(n=3)$, ischaemic heart disease $(n=1)$, poorly controlled diabetes and being morbidly obese $(n=2)$, high risk of disease recurrence $(n=1)$, poor functional status / reduced mobility $(n=2)$, tumour progression ( $n=2)$ and lack of anticipated impact on quality of life $(n=4)$. Thirty-six percent $(n=8)$ of the patients not listed for transplant died a mean of $3.5 \pm 3.5$ years after the date of their initial transplant assessment. Seven of those who died were patients deemed unfit to be listed for transplantation.

Follow-up data were available for 72 of the 81 patients for a median of 4.6 years (IQR 2.3-7.0) post initial transplant assessment. Fig 3 illustrates the difference in survival between those patients who did and did not undergo OLT. Patients who underwent OLT had a significantly longer mean survival time of 


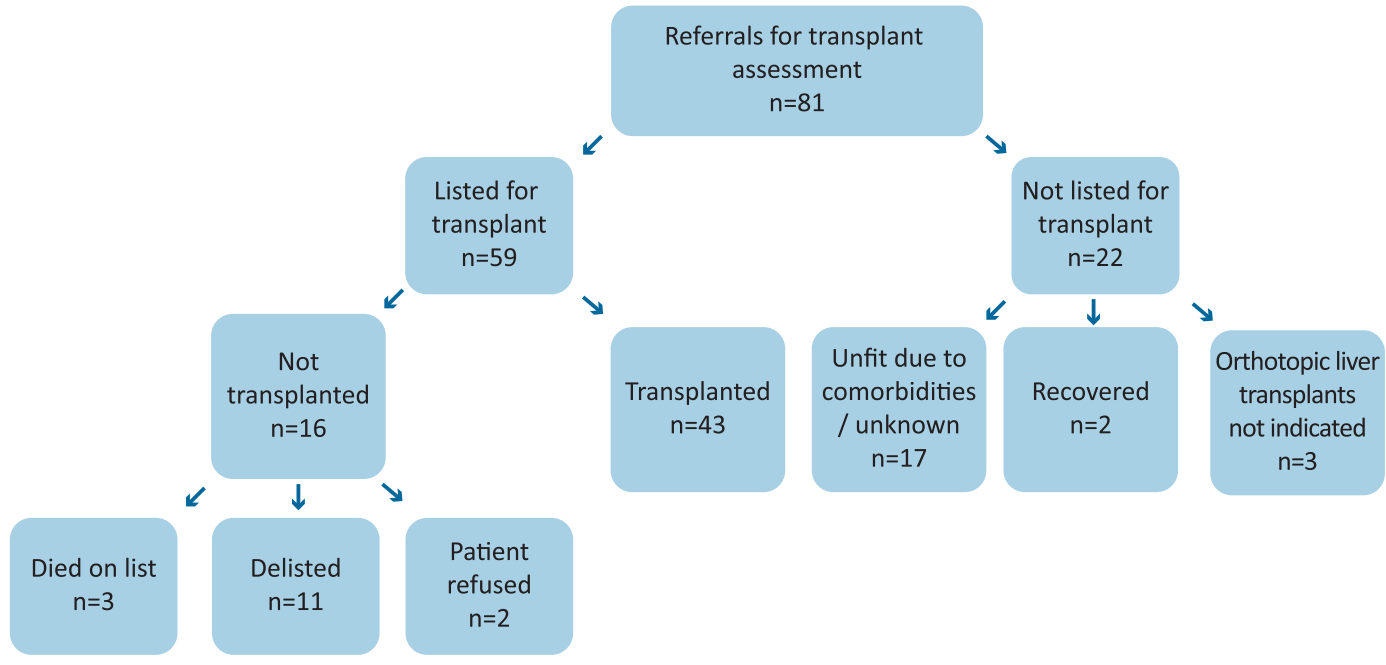

Fig 2. Outcomes of transplant assessment.

9.81 years $(95 \%$ confidence interval (CI) 8.51-11.12) compared with those who did not undergo OLT (mean survival time of 4.62 years; $95 \%$ CI 3.35-5.89; $p<0.001$ ). There was no significant difference in the survival time between those presenting to secondary care with (mean 7.63 years; 95\% CI 6.32-8.95) or without (mean 6.74 years; $95 \%$ CI 5.07-8.41) a primary decompensating event $(p=0.263)$.

\section{Discussion}

The indolent and asymptomatic nature of NAFLD makes identification of patients with cirrhosis in whom specific medical or lifestyle interventions might ameliorate their condition a significant clinical challenge. ${ }^{17}$ The prevalence of liver related deaths in the UK continue to rise in comparison with other major causes of death and NAFLD makes an increasing contribution to this mortality. ${ }^{18-22}$

Previous studies have highlighted that relying upon clinical detection of advanced fibrosis in NAFLD is an inadequate strategy, as the absence of symptoms and poor sensitivity of tests leads to late diagnosis. ${ }^{23}$ This finding is supported by our data, as although $100 \%$ of the patients had established cirrhosis at the time of their first presentation to healthcare, this was not recognised in $24.7 \%$ of patients who underwent OLT assessment.

An Australian survey assessing primary care practitioners' knowledge of NAFLD highlighted that the majority of respondents underestimated the prevalence of NAFLD in the general population. ${ }^{24}$ They also identified that over $70 \%$ of
Fig 3. Survival curve analysis for patients who underwent orthotopic liver transplant.

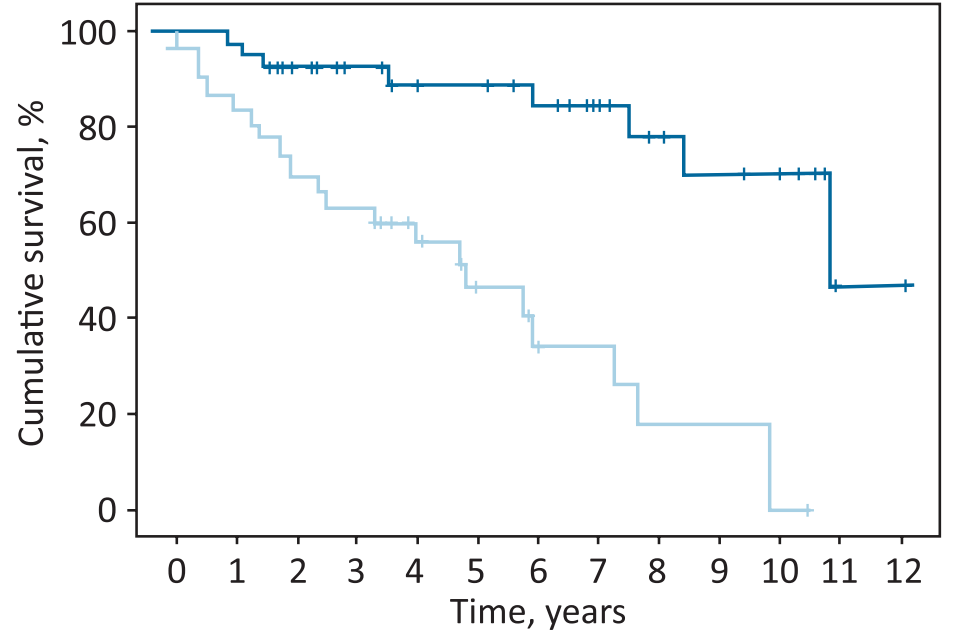

Not transplanted $\begin{array}{lllllllllllll}31 & 25 & 21 & 19 & 13 & 8 & 5 & 4 & 2 & 2 & 1 & 0 & 0\end{array}$ $\neg$ Transplanted $\begin{array}{lllllllllllll}41 & 40 & 33 & 27 & 23 & 22 & 19 & 15 & 11 & 9 & 8 & 1 & 1\end{array}$

At risk, $n$ 
practitioners were unlikely to refer for a specialist opinion unless liver function tests were abnormal. Our data support this finding, as 18 patients ( $35 \%$ of primary care referrals) were referred for investigation of abnormal LFTs without recognition that these patients had already developed cirrhosis. Supporting this, recent clinical guidelines have emphasised the use of fibrosis algorithms or serum biomarkers as opposed to routine liver blood tests in identifying advanced fibrosis in patients with NAFLD. ${ }^{2}$

Other studies have illustrated that the presence of decompensated NAFLD at initial presentation to healthcare is associated with worse outcomes. ${ }^{25}$ Of concern, our data demonstrate that the majority of patients $(64.0 \%)$ undergoing OLT assessment for NAFLD already had decompensated cirrhosis at the time of their first diagnosis of CLD. In addition, the majority $(91.7 \%)$ of emergency admissions to hospital had decompensated disease at the time of their first presentation. These data undoubtedly underestimate the mortality associated with NAFLD cirrhosis, as this study did not investigate how many patients presenting with decompensated NAFLD cirrhosis died before being considered for liver transplantation.

These late presentations of NAFLD cirrhosis illustrate the need for more effective strategies to ensure that patients with NAFLD fibrosis are identified in primary care. Guidelines from the European Association for the Study of the Liver (EASL), the European Association for the Study of Diabetes (EASD) and the European Association for the Study of Obesity (EASO) have suggested targeted stepwise screening for NAFLD in high-risk groups to identify the 'at risk' population advising early referral to optimise management. ${ }^{16}$ However, a recent review questions this approach, reminding us that the patients at risk of liver-related mortality are those with advanced fibrosis and cirrhosis (F3/F4 fibrosis), and those with F0-F2 fibrosis do not require liver specific care. ${ }^{26}$ Although patients without advanced fibrosis do not require specialist hepatology review, identification of these patients to optimise their modifiable risk factors, such as obesity and diabetes, can lead to fibrosis regression and prevent progression of disease, potentially lessening the burden on hepatology service. ${ }^{9}$ The recent National Institute for Health and Care Excellence guidance on the management of NAFLD goes some way to address both of these issues by emphasising the risks of liver disease in obese patients and in recommending the use of non-invasive testing for liver fibrosis through use of the enhanced liver fibrosis (ELF) test, a simple serological test that can be incorporated alongside routine blood tests in those at risk of NAFLD. ${ }^{2}$ A recent UK study prospectively assessing the use of the ELF test as part of a twostep algorithm identified that use of the algorithm had a five-fold increase in detection of $\mathrm{F} 3 / \mathrm{F} 4$ fibrosis and an $88 \%$ reduction in inappropriate referrals. ${ }^{27}$

Although 59 patients were placed on the waiting list for transplantation, only 43 underwent transplantation. Seventy per cent of those transplanted presented with decompensated disease. Deaths in the transplanted patients $(n=4)$ in the perioperative period were limited to those presenting to secondary care with decompensated NAFLD. The most common reason for not listing patients was due to comorbidities related to obesity and the metabolic syndrome. These data demonstrate the poor general condition of many patients with NAFLD at the time of transplant assessment and illustrate that NAFLD is part of a multisystem disorder. Greater awareness of NAFLD and the multimorbidity associated with the metabolic syndrome would enable primary care physicians and other healthcare practitioners to address the other pathologies associated with NAFLD as well as investigating liver disease.

\section{Study limitations}

This retrospective cohort study was limited to those patients formally assessed for liver transplantation and is thus subject to the biases associated with all studies conducted in transplant centres. We were unable to quantify the number of patients never referred to tertiary care for OLT assessment due to comorbidities and those who died before OLT assessment, potentially underestimating the morbidity and mortality of NAFLD cirrhosis. In contrast, the impact of NAFLD cirrhosis may have been exaggerated in our study by omission of cirrhotic patients with a benign disease course, including those patients in whom successful interventions mitigated the need for OLT.

It is beyond the scope of this study to determine the outcomes of patients with NAFLD not referred for transplant assessment. The natural history of NAFLD is reviewed elsewhere. ${ }^{28}$ Rather we have focused on the epidemiology of patients referred to our service with advanced disease. Due to the retrospective nature of the data collection, we were unable to accurately identify whether patients were offered prehabilitation prior to listing. While this would have been recommended, there was no formal prehabilitation programme at this time. Although alcohol histories were not formally recorded, all patients undergoing transplant listing with a diagnosis of NAFLD had alcohol excluded as a cause of their cirrhosis and all were abstinent from the time of listing. Due to the prolonged study period, there was some variation in the assessment of patients' frailty and fitness to undergo transplantation.

This study considered patients in whom NAFLD was the sole indication for transplantation and thus patients with mixed aetiologies of CLD were excluded. Furthermore, the study period encompassed the years when NAFLD emerged as a diagnostic category and so the cohort reflects the emergence of NAFLD as a recognised entity and may not be representative of current referral practice.

\section{Conclusion}

All of the patients who underwent assessment for OLT with NAFLD as the primary indication had cirrhosis at the time of their first presentation to healthcare. This diagnosis had not been recognised in a quarter of those referred. The majority of patients had evidence of decompensated cirrhosis at their first presentation to secondary care. These cases of late diagnosis represent missed opportunities for earlier intervention to prevent disease progression. Earlier identification and appropriate treatment of signs and symptoms of CLD prior to decompensation, especially with new and emerging treatments of NAFLD, may improve clinical outcomes and patient quality of life.

\section{Funding}

This work was funded by a National Institute for Health Research Senior Investigator award received by Prof Rosenberg. 


\section{Supplementary material}

Additional supplementary material may be found in the online version of this article at www.rcpjournals.org/clinmedicine:

S1 - Patient journey to orthotopic liver transplant assessment clinic at a tertiary transplant centre.

S2 - Index presentation of chronic liver disease in patients undergoing orthotopic liver transplant assessment $(n=81)$. S3 - Reasons for referral to a level 3 hepatology centre.

\section{References}

1 Dyson JK, Anstee QM, McPherson S. Non-alcoholic fatty liver disease: a practical approach to treatment. Frontline Gastroenterol 2014;5:277-86.

2 Glen J, Floros L, Day C et al. Non-alcoholic fatty liver disease (NAFLD): summary of NICE guidance. BMJ 2016;354:14428.

3 Lonardo A, Sookoian S, Pirola C] et al. Non-alcoholic fatty liver disease and risk of cardiovascular disease. Metabolism 2016;65:1136-50.

4 Ekstedt M, Hagstrom H, Nasr P et al. Fibrosis stage is the strongest predictor for disease-specific mortality in NAFLD after up to 33 years of follow-up. Hepatology 2015;61:1547-54.

5 Younossi ZM, Stepanova M, Rafiq N et al. Pathologic criteria for nonalcoholic steatohepatitis: interprotocol agreement and ability to predict liver-related mortality. Hepatology 2011;53:1874-82.

6 Cholankeril G, Wong RJ, Hu M et al. Liver transplantation for nonalcoholic steatohepatitis in the US: Temporal trends and outcomes. Dig Dis Sci 2017;62:2915-22.

7 NHS Blood and Transplant. Annual report on liver transplantation: Report for 2018/2019. NHS, 2019. https://nhsbtdbe.blob.core.windows.net/umbraco-assets-corp/16782/nhsbt-liver-transplantationannual-report-2018-19.pdf.

8 Garbuzenko DV, Arefyev NO. Current approaches to the management of patients with cirrhotic ascites. World J Gastroenterol 2019;25:3738-52.

9 Vilar-Gomez E, Martinez-Perez Y, Calzadilla-Bertot L et al. Weight loss through lifestyle modification significantly reduces features of nonalcoholic steatohepatitis. Gastroenterology 2015;149:367-78. e5; quiz e14-5.

10 Okuda K, Ohtsuki T, Obata H et al. Natural history of hepatocellular carcinoma and prognosis in relation to treatment. Study of 850 patients. Cancer 1985;56:918-28.

11 The Cancer of the Liver Italian Program (CLIP) investigators. A new prognostic system for hepatocellular carcinoma: a retrospective study of 435 patients. Hepatology 1998;28:751-5.

12 Llovet JM, Bustamante J, Castells A et al. Natural history of untreated nonsurgical hepatocellular carcinoma: rationale for the design and evaluation of therapeutic trials. Hepatology 1999;29:62-7.

13 Villa E, Moles A, Ferretti I et al. Natural history of inoperable hepatocellular carcinoma: estrogen receptors' status in the tumor is the strongest prognostic factor for survival. Hepatology 2000;32:233-8.
14 Cabibbo G, Enea M, Attanasio M et al. A meta-analysis of survival rates of untreated patients in randomized clinical trials of hepatocellular carcinoma. Hepatology 2010;51:1274-83.

15 Blais P, Husain N, Kramer JR et al. Nonalcoholic fatty liver disease is underrecognized in the primary care setting. Am J Gastroenterol 2015;110:10-4.

16 European Association for the Study of the Liver (EASL), European Association for the Study of Diabetes (EASD), European Association for the Study of Obesity (EASO). EASL-EASD-EASO Clinical Practice Guidelines for the management of non-alcoholic fatty liver disease. J Hepatol 2016;64:1388-402.

17 Armstrong MJ, Houlihan DD, Bentham L et al. Presence and severity of non-alcoholic fatty liver disease in a large prospective primary care cohort. J Hepatol 2012:56:234-40.

18 Murray CJ, Richards MA, Newton JN et al. UK health performance: findings of the Global Burden of Disease Study 2010. Lancet 2013;381:997-1020.

19 Bhala N, Aithal G, Ferguson J. How to tackle rising rates of liver disease in the UK. BMJ 2013;346:f807.

20 Adam R, Karam V, Delvart V et al. Evolution of indications and results of liver transplantation in Europe. A report from the European Liver Transplant Registry (ELTR). J Hepatol 2012;57: 675-88.

21 Bertuccio P, Turati F, Carioli G et al. Global trends and predictions in hepatocellular carcinoma mortality. J Hepatol 2017;67:302-9.

22 Wild SH, Morling JR, McAllister DA et al. Type 2 diabetes and risk of hospital admission or death for chronic liver diseases. $]$ Hepatol 2016;64:1358-64.

23 Harman DJ, Ryder SD, James MW et al. Direct targeting of risk factors significantly increases the detection of liver cirrhosis in primary care: a cross-sectional diagnostic study utilising transient elastography. BMJ Open 2015;5:e007516.

24 Patel PJ, Banh X, Horsfall LU et al. Underappreciation of non-alcoholic fatty liver disease by primary care clinicians: limited awareness of surrogate markers of fibrosis. Internal medicine journal 2018;48:144-51.

25 Ratib S, Fleming KM, Crooks C] et al. 1 and 5 year survival estimates for people with cirrhosis of the liver in England, 1998-2009: a large population study. J Hepatol 2014;60:282-9.

26 Rowe IA, Parker R. The diagnosis of nonalcoholic fatty liver disease should carry important prognostic information. Nat Rev Gastroenterol Hepatol 2019;16:449-50.

27 Srivastava A, Gailer R, Tanwar S et al. Prospective evaluation of a primary care referral pathway for patients with non-alcoholic fatty liver disease. J Hepatol 2019;71:371-8.

28 Ekstedt M, Nasr P, Kechagias S. Natural History of NAFLD/NASH. Curr Hepatol Rep 2017;16:391-7.

Address for correspondence: Prof William Rosenberg, Institute for Liver and Digestive Health, UCL Medical School, Royal Free Hospital, Pond Street, London NW3 2PF, UK. Email:w.rosenberg@ucl.ac.uk 